\title{
Intracoronal stress transfer through enamel following RBC photopolymerisation: A synchrotron X-ray study
}

Maisoon Al-Jawad ${ }^{\mathrm{a}, * *}$, Owen Addison ${ }^{\mathrm{b}, \mathrm{c}, *}$, Slobodan Sirovicac, ${ }^{\mathrm{d}}$, Samera Siddiqui ${ }^{\mathrm{a}}$, Richard A. Martin ${ }^{\mathrm{d}}$, David J. Wood ${ }^{\mathrm{e}}$, David C. Watts ${ }^{\mathrm{f}}$

a Institute of Dentistry, Barts and The London School of Medicine and Dentistry, Queen Mary University of London,

London, UK

b Biomaterials Unit, University of Birmingham School of Dentistry, Birmingham, UK

c University of Alberta, School of Dentistry, Edmonton, AB, Canada

d Aston Institute of Materials Research, Aston University, Birmingham, UK

e Biomaterials and Tissue Engineering Research Group, School of Dentistry, University of Leeds, Leeds, UK

f School of Medical Sciences and Photon Science Institute, University of Manchester, UK 


\section{Abstract}

Objectives: To measure the spatial distribution of crystallographic strain in tooth enamel induced by the photo-polymerisation of a dimethacrylate resin based composite cavity restoration.

Methods: Six sound first premolar teeth, allocated into two groups $(n=3)$, were prepared with mesioocclusal distal cavities. The enamel was machined at the point of maximum convexity on the outer tooth to create a vertical fin of thickness $100 \mu \mathrm{m}$ and $0.5 \mathrm{~mm}$ depth to allow for synchrotron X-ray diffraction measurements. 2D diffraction patterns were used to determine crystallite orientation and quantify changes in the hydroxyapatite crystal lattice parameters, before and after photo-polymerisation of a composite material placed in the cavity, to calculate strain in the respective axis. The composite was photo-polymerised with either relatively high $\left(1200 \mathrm{mWcm}^{-2}\right.$, group 1$)$ or low $\left(480 \mathrm{mWcm}^{-2}\right.$, group 2$)$ irradiances using LED or quartz halogen light sources, respectively. A paired t-test was used to determine significant differences in strain between irradiance protocols at $\mathrm{a}=0.001$.

Results: Photo-polymerisation of the composite in the adjacent cavity induced significant changes in both the crystallographic $c$ and $a$ axes of the enamel measurement area. However the magnitude of strain was low with $\sim 0.1 \%$ difference before and after composite photo-polymerisation. Strain in enamel was not uniformly distributed and varied spatially as a function of crystallite orientation. Increased alignment of crystallites perpendicular to the cavity wall was associated with higher $c$ axis strain. Additionally, strain was significantly greater in the $c(\mathrm{p}<0.001)$ and $a$ axis $(\mathrm{p}<0.001)$ when using a high irradiance photo-polymerisation protocol.

Significance: Although cuspal deflection is routinely measured to indirectly assess the 'global' effect of composite shrinkage on the tooth-restoration complex, here we show that absolute strains generated in enamel are low, indicating strain relief mechanisms may be operative. The use of low irradiance protocols for photo-polymerisation resulted in reduced strain. 


\section{Introduction}

Dental resin based composite materials exhibit a volumetric shrinkage associated with polymerisation that, when constrained by adhesion to the tooth cavity walls, leads to the generation of shrinkage stresses within the tooth-composite complex. Shrinkage stresses have been measured directly in-vitro and it has been identified that the magnitude and kinetics of their development are dependent on a number of factors, including in particular, the composite composition and resin chemistry $[1,2]$, photopolymerisation variables [3-5] and the terminal degree of monomer to polymer conversion [6-8]. It has been proposed that both the magnitude of the shrinkage stress, and the kinetics of its development, are important factors in determining whether shrinkage stresses have an unfavourable impact on the remaining tooth tissue or on the adhesive interface between the composite and enamel and dentine. Stress transfer to the adhered tooth complex has been directly demonstrated using cuspal deflection methods and indirectly indicated through micro-leakage measurements [9-14] which are proposed to reflect interfacial debonding that has occurred as a stress relief mechanism [15-18]. Shrinkage stresses arise because the volumetric free shrinkage of the polymer matrix of composites that occurs during polymerisation is constrained by the geometrical confines of the host tissue substrate to which it is adhered [19-21]. It is accepted that the magnitude of stress generated is influenced by the monomer composition [1, 5, 22, 23], the polymerisation rate [24-28], the stiffness of the polymerised cross-linked matrix and the nature of the external constraints [29-34], which can also be considered as the compliance of the system [35, 36]. Of these factors, the effects of resin-matrix monomer composition, polymerisation kinetics and subsequent mechanical properties of the composite including its adhesion to the tooth substrate have arguably been disproportionately studied, when compared with understanding stress transfer to the tooth and potential stress relief mechanisms within the toothrestoration complex.

The tooth is a structure comprised of a highly mineralised (94-96 wt\% hydroxyapatite $[37,38])$ thin surface layer of enamel which is supported by a relatively compliant, less mineralised ( $70 \mathrm{wt} \%$ ) dentine substrate [39]. At the interface, adhesion between enamel and dentine is manifested by a hierarchically scalloped topology and a relatively protein rich organic matrix [40-43]. Enamel and dentine and their interface are subjected to extreme cyclic mechanical and thermal stresses. However, 
they show remarkable resistance to mechanical failure $[44,45]$. Notably enamel has no cellular mediated capacity for repair but it is proposed that its hierarchical structure provides an inherent ability for stress relief conferring a damage tolerance that enables it to survive for the lifespan of the host [46, 47]. Enamel structure is comprised of nanoscale hydroxyapatite needle-like crystallites that are specifically orientated to form microscale 'keyhole shaped' prisms [38, 39, 48, 49], that are separated from each other by less organised, protein rich interfaces [50-52]. Both hydroxyapatite crystallites and enamel prisms have distinct orientations that are a function of their location [53-55]. As a bulk material, enamel is anisotropic with respect to its elastic modulus [56], exhibits a high fracture toughness [57] and has been shown to exhibit time dependent deformation as a response to applied stress. Using nanoindentation methods it has been shown that the creep behaviour of enamel was more similar to metallic materials than to its hydroxyapatite constituent [38]. These responses were attributed to the organic protein content, found between hydroxyapatite crystals and between prisms, which permitted both inelastic deformation and subsequent partial recovery of the deformation $[58,59]$.

The capacity for stress relief within the enamel structure may go some way to explaining the inconsistencies in data generated from the cuspal deflection method [27, 28, 60-63] using natural teeth. Cuspal deflection measurements typically require a large mesio-occlusal-distal (MOD) cavity to be prepared in a tooth and the distance between the points of maximum convexity of the facial and lingual surfaces to be recorded dynamically during composite placement and photo-polymerisation [32-34, 36, 64]. The magnitude of the decrease in inter-cuspal distance is used as a surrogate outcome of the net shrinkage stress generated and distributed within the tooth-composite complex. Such measurements cannot discriminate the vector and location of stress generation and indeed any stress relief that occurs, within the tooth, the composite or the interface between materials. A number of cuspal deflection studies have failed to show differences between variables that were strongly assumed to generate different magnitudes of shrinkage stress [62, 63].

The aim of this study was for the first time to measure strains within the enamel that had been induced by the polymerisation of dental composites. Using two-dimensional synchrotron X-ray diffraction (2DSXRD) it is possible to measure simultaneously lattice strain and crystallite organisation by observing the changes in lattice parameters and preferred orientation of the crystalline phase (hydroxyapatite). 
The specific objective was to quantify the spatial distribution of strain generated in the hydroxyapatite lattice of human dental enamel. Photo-polymerisation of a single composite material was induced using two different irradiances, nominally considered as 'high' and 'low' to generate differences in the kinetics of shrinkage stress generation $[7,65]$. 


\section{Materials and Methods}

\subsection{Sample Preparation}

Unrestored human maxillary first premolars of similar size and morphology, previously extracted for orthodontic purposes, were selected from a tissue bank (UK ethical approval, NRES-14/EM/2811, University of Birmingham, UK). Teeth were inspected using light microscopy and included if they were caries free, exhibited no enamel cracks or other defects and had buccal palatal widths of $8.5 \pm 0.1 \mathrm{~mm}$ measured with a digital micrometer. MOD cavities were cut using an air turbine with a pattern 541 diamond bur (MDT technologies, Israel) under copious water irrigation according to the protocol described by Kearns et al [12]. Briefly, the proximal boxes were prepared to extend vertically to $1 \mathrm{~mm}$ above the cemento enamel junction (CEJ) with a width of two thirds of the buccal palatal width. The occlusal isthmus dimensions were standardised with a $3.5 \mathrm{~mm}$ depth relative to the palatal cusp and a width of half the buccal palatal width.

To provide a standardised thickness of facial enamel for micro-focussed X-ray diffraction measurements, the buccal enamel surface of each tooth was further machined using an air turbine with a 541 pattern diamond bur under water irrigation. Using an operating microscope, the enamel was machined at the point of maximum convexity to create a vertical 'fin' (Figure 1) of $0.5 \mathrm{~mm}$ depth and $100 \mu \mathrm{m}$ thickness (measured using a digital micrometer). Teeth were stored in moist conditions at $4 \pm$ $1{ }^{\circ} \mathrm{C}$ prior to further use.

\subsection{Synchrotron X-ray Diffraction Mapping}

Synchrotron X-ray diffraction (SXRD) measurements were carried out on the B16 beamline [66] at the Diamond Light Source (Oxford Harwell Campus, Didcot, UK). An incident X-ray energy of $20 \mathrm{keV}$ was used, equivalent to a wavelength $(\lambda)$ of $0.6212 \AA$, with a beam size of $100 \mu \mathrm{m} \times 100 \mu \mathrm{m}$ defined using a compound refractive lens. Samples were mounted normal to the impinging X-rays in transmission geometry onto a travelling $\mathrm{x}-\mathrm{y}$ sample stage to allow measurements in two orthogonal directions perpendicular to the X-ray beam. A single SXRD measurement had an exposure time of 180 s and was collected using a 2D area detector (Image Star 9000, Photonic Science Ltd. UK) [67] with a 
$3056 \times 3056$ pixel resolution $(31 \times 31 \mu \mathrm{m}$ optical pixel size)[67], placed $155 \mathrm{~mm}$ behind the sample to give a $2 \theta$ range of $5-25^{\circ}$. Instrument parameters including the X-ray wavelength and experimental geometry were accurately determined by calibrating using lanthanum hexaboride $\left(\mathrm{LaB}_{6}\right)$ (Sigma Aldrich, Dorset, UK) standard samples.

Due to the precision required for repeat measurements of the identical areas of enamel before and after photo-polymerisation, the MOD cavities were bulk-filled with composite in dark conditions prior to all measurements to avoid any possible sample movement. In advance, the peripheral enamel of each prepared tooth sample was etched for $30 \mathrm{~s}$ and dentine was etched for $15 \mathrm{~s}$ with $35 \%$ phosphoric acid before thorough rinsing in distilled water. After gentle air drying, an adhesive (Single Bond Universal, 3M ESPE, MN, USA) was applied according to manufacturer's instructions and photo-polymerised from the occlusal and proximal aspects for 20s each. The tooth was left in air for $>6 \mathrm{~h}$ to allow for dehydration which may effect to sample stability during measurements to occur before composite placement. Subsequently the cavity was bulk-filled with a commercial composite resin (Figure 1) (Z100" ${ }^{\mathrm{TM}}$ MP Universal Composite, 3M ESPE, MN, USA) based on bisphenol-A-glycidyl-methacrylate (Bis-GMA) and triethyleneglycol-dimethacrylate (TEGDMA) monomers and utilising a Camphorquinone / tertiary amine photo-initiator system. The composite was shielded from light with aluminium foil prior to the tooth being mounted rigidly on an x-y stage. SXRD measurements were taken from the top to the bottom of each tooth 'fin' in successive tracks, starting closest to the cavity wall with up to four tracks used to spatially characterise crystallographic changes. The vertical step size between SXRD measurements along the same track was $0.2 \mathrm{~mm}$, whilst adjacent tracks were separated horizontally by $0.1 \mathrm{~mm}$. Due to the time constraints of using a synchrotron source only two of the six tooth specimens were mapped over a larger area consisting of four tracks (Figure 1), whilst the remaining samples were mapped with a single track. Larger area maps were sacrificed to gain repeat measurements for statistical analysis. All measurements were conducted in dark conditions at $23 \pm 1{ }^{\circ} \mathrm{C}$.

Following initial mapping of the unstrained tooth specimen, the aluminium shielding was removed and any minor slumping of the composite material at the mesial and distal boxes was corrected using gentle manual adjustments. Two light curing units (LCUs) providing relative high and low light irradiances 
were used for photo-polymerisation respectively. To provide a relative high irradiance, the composite was cured using an LED LCU (Elipar S10, 3M ESPE, MN, USA) with an irradiance of $1200 \mathrm{mWcm}^{-2}$ over a spectral emission range of 430 - $480 \mathrm{~nm}$. To produce a lower irradiance, a quartz tungsten halogen LCU (Optilux 501, Kerr dental, CA, USA) with an irradiance of $480 \mathrm{mWcm}^{-2}$ over a spectral emission range of 400 - $505 \mathrm{~nm}$ was used. A $13 \mathrm{~mm}$ light guide was used for both LCUs and the composite was illuminated for $60 \mathrm{~s}$ from the occlusal surface at a distance of $2 \mathrm{~mm}$ followed by a $30 \mathrm{~s}$ exposure each on the mesial and distal aspects from $0 \mathrm{~mm}$.

Following a fixed time of $30 \mathrm{~min}$ after polymerisation, to allow for post-cure effects, SXRD measurements were repeated across the same track positions under identical conditions as previously described. The process of initial map measurement, composite application/polymerisation and remapping was repeated three times per photo-polymerisation regimen on separate (unstrained) tooth specimens generating measurements for a total of six teeth with three teeth per curing protocol. Repeat measurements allowed for the spatial verification of relative changes in the crystallographic structure and statistical analysis for relevant lattice parameters for each curing regimen.

\subsection{Data Processing and Analysis}

Two-dimensional diffraction images were processed using the ESRF software package Fit2D (version 12.077, ESRF)[68]. Diffraction patterns were azimuthally integrated to produce 1D spectra of intensity (I) versus the scattering angle $(2 \theta)$. The Bragg peaks corresponding to the $(002)\left(\sim 10^{\circ}\right)$ and $(300)\left(\sim 13^{\circ}\right)$ Bragg reflections were fitted with pseudo-Voigt peak shapes to obtain the peak position and the full width at half maximum (FWHM) to quantify possible changes to the crystal structure from the lattice parameters and peak shape. The (002) and (300) planes were used to measure relative changes in the lattice parameters as they are normal to the $c$ and $a$ axes of the hydroxyapatite unit cell respectively. Peak positions were used to determine the inter-planar spacing $(d)$, where $d=\lambda / 2 \sin \theta$, from which lattice parameters were calculated. A hexagonal unit cell was assumed for hydroxyapatite, where the relationship between the $d$ spacing and Miller indices for a given Bragg peak and lattice parameters is given as; 


$$
\frac{1}{d^{2}}=\frac{4}{3}\left(\frac{h^{2}+h k+k^{2}}{a^{2}}\right)+\frac{l^{2}}{c^{2}}
$$

where $h, k$ and $l$ refer to the Miller indices for a particular Bragg peak whilst $a$ and $c$ represent the relevant lattice parameters. Substituting the (002) and (300) indices into equation (1) gives the individual $c$ and $a$ lattice parameters respectively as a function of $d$.

$$
\begin{gathered}
c=2 d \\
a=\sqrt{12} d
\end{gathered}
$$

Therefore, any crystallographic strain generated in the tooth associated with composite photopolymerisation was calculated by precisely measuring changes in the $c$ and $a$ lattice parameters at identical locations before and after photo-curing. Lattice strain $(\xi)$ was calculated for every SXRD measurement point along each track to produce strain maps, where the lattice strain was given as the percentage change in the lattice parameters, as shown in equation (4),

$$
\xi=\left(\frac{P_{\text {after }}-P_{\text {before }}}{P_{\text {before }}}\right) \times 100
$$

Here $P_{\text {before }}$ and $P_{\text {after }}$ refer to the value of a given lattice parameter as measured at each point before and after the application of the resin composite. Strain maps across the tooth 'fins' were created from these values. For each photo-polymerisation regimen a paired t-test was used to test for statistical significance of the changes observed in the $a$ and $c$ axis lengths ( $\mathrm{a}=0.001)$. All calculations were performed using $\mathrm{R}$ programming (version 3.1.3, 2015, R Foundation).

Texture parameters, including the direction and magnitude of the preferred orientation were obtained by integrating the intensity around the Debye ring of the (002) Bragg reflection over $360^{\circ}$ and plotted against the azimuthal angle. Preferred orientation was determined as this reflection is perpendicular to the $c$ axis of the hydroxyapatite unit cell. Peaks were fitted with a Gaussian shape to calculate the 
deviation angle $\varphi$ of the crystallite axis with respect to the vertical axis and to also obtain the full width at half maximum (FWHM). The FWHM of the azimuthally integrated peaks gives a measure of the crystallite alignment (or preferred orientation) where high FWHM values indicate lower alignment and low FWHM values indicate higher alignment [69].

\section{Results}

\subsection{Crystallite Orientation Direction and Magnitude}

Figure 2 shows typical synchrotron X-ray diffraction (SXRD) results for the tooth specimens measured. 2D diffraction patterns collected on the area detector are shown in Figures $2 \mathbf{a}$ and $\mathbf{2 b}$ and a radially integrated plot, showing characteristic diffraction peaks typical of hydroxyapatite, is given in Figure 2c. The $(h k l)$ indices used for the study of crystallographic strain were the (002) and (300) Bragg reflections that are labelled in Figure 2c. It can be seen in Figure 2a that the intensity of the (002) Debye ring varies as a function of the azimuthal angle. This is indicative of texture (preferred orientation) within the hydroxyapatite phase of the dental enamel. The strongest texture was found in the (002) diffraction ring, and the direction of the crystallite alignment is marked in Figure 2a (blue line). Thus the orientation direction and magnitude of alignment of the needle-like "nanorod" crystallites of enamel can be quantified by analysis of the 2D diffraction patterns. In comparison, Figure 2b shows a complete (002) diffraction ring, demonstrating little or negligible texture.

In general, it was observed that the orientation of the nanorods is approximately perpendicular to the cavity wall as illustrated in Figure 3a (red markers). Assuming a hexagonal unit cell, the $a=b$ axis will therefore run perpendicular to the $c$ axis and approximately parallel to the cavity wall. Azimuthal integration of the (002) reflection yielded a bimodal distribution (Figure 3b) for each intensity arc, with two maxima located at $\sim 180$ and $210^{\circ}$ to the cavity wall respectively. This feature of two distinct populations of crystallite orientations was present in all tooth samples prior to polymerisation and remains unaltered by the photo-polymerisation of a composite. The direction of orientation for each population was obtained by the deconvolution of the peaks with a Gaussian profile. The integrated peak intensity gave the proportion of crystallites and/or enamel prisms belonging to each population. Typically, $75-90 \%$ of the crystallites (of the total integrated peak intensities) displayed a $c$-axis preferred 
orientation at an approximately perpendicular angle $\left(\sim 180^{\circ}\right)$, normal to the cavity wall, however this proportion varies spatially within each sample. The non-perpendicular population of crystallites exhibit an orientation at a more acute angle to the cavity wall at $\sim 210^{\circ}$ (i.e. with an angular separation from the other population of approximately $30^{\circ}$ ).

\subsection{Crystallographic Strain}

Figure 4 illustrates the typical spatial distribution of the $c$ lattice parameter and $\%$ strain in the $c$-axis calculated using equations (1), (2), and (4) and reconstructed as colour contour maps, over the scanned region of a given enamel 'fin' before and after the application of composite for relatively 'fast' and 'slow' polymerisation rates. Prior to composite photo-polymerisation the unstrained enamel had mean $c$ lattice parameters of $6.91 \pm 0.01 \AA$ and $6.85 \pm 0.01 \AA$ for the 'high' and 'low' irradiance groups respectively ( $n=3$ per group). Figures $4 \mathbf{b}$ and $4 \mathbf{e}$ shows maps of the $c$ lattice parameter following polymerisation of the composite in the cavity using the LED and QTH light sources, respectively. The spatial distribution of the $c$ lattice parameter appears similar to the unstrained case for both curing protocols, however the absolute values of $c$ axis length (a measure of crystallographic lattice strain introduced into the unit cell) decreased in both cases (Figures $\mathbf{4 c}$ and $\mathbf{f}$ ). The composite polymerised using the higher irradiance (LED) induced strain in the $c$ lattice axis of the adjacent enamel approximately 2-5 times greater than the low irradiance (QTH) regimen. This was consistent across all repeat measurements in all specimens measured, where mean values across all specimens were $-0.12 \pm$ 0.03 and $-0.053 \pm 0.006 \%$ for the high and low irradiance protocols respectively. For both irradiances, the spatial distribution of strain exhibited a slight vertical gradient with increasing strain values towards the bottom of the enamel fin, parallel to the cavity base (Figures $\mathbf{4 c}$ and $\mathbf{f}$ ). The magnitude of strains generated in the $a$ lattice axis were less than those observed for the $c$ axis with mean values given as $0.104 \pm 0.005 \%$ and $0.002 \pm 0.006 \%$ for the high and low irradiance protocols, respectively. No clear pattern in the distribution of the magnitude of strain in the $a$ axis was observed. When compared with the $c$ axis, the magnitude of strain was more consistent across the measurement area (Figure 5). Paired t-tests confirmed that photo-polymerisation resulted in significant changes in the $c$ axis length for both curing regimens $(\mathrm{p}<0.001)$ whereas only the high irradiance regimen resulted in a significant change in the $a$ lattice parameter (Figure 5). 


\subsection{Impact of Crystallite Orientation on Resultant Strain}

Azimuthal integration of the (002) diffraction ring revealed a bimodal distribution with respect to crystallite orientation (Figure 3b) with two distinct populations of crystallites observed, with preferred orientation directions of $\sim 180^{\circ}$ and $\sim 210^{\circ}$ in the azimuthal plane, that we have labelled the perpendicular and non-perpendicular angle populations, respectively. The analysis described in section 3.1 was performed on the SXRD images prior to and post polymerisation to ascertain any effects that composite polymerisation had on crystallite orientation. Figure 6 shows the post-polymerisation change in the azimuthal angle for the perpendicular (blue) and non-perpendicular angle (red) crystallite populations as a function of distance from the top of the enamel fin along the track nearest to the cavity for the high irradiance protocol (LED) sample. The strain in the $c$ lattice is also plotted in Figure 6 (black). There was no significant correlation between the changes in orientation and $c$ axis strain along individual measurement tracks, with Pearson correlation coefficients of $r=0.210$ and $r=-0.038$ for the perpendicular angle and non-perpendicular angle crystallite orientation populations. Figure 7 illustrates the spatial relationship between the bimodal orientation of the hydroxyapatite crystallites and the $a$ and $c$ axis strain distributions respectively in the tooth fin for all four tracks. The direction of the perpendicular and non-perpendicular orientations (with respect to the cavity wall) are plotted as blue and red bars respectively. The length of the bar indicates the proportion of crystallites belonging to each population with the perpendicular population the more dominant and therefore represented as a longer blue bar in Figure 7. Bar lengths are scaled to the integrated intensity of the respective peak and normalised to the total integrated intensity. Orientation bars are superimposed over the strain colour map with the red bars scaled in length by a factor of three and the aspect ratio of the underlying strain maps altered for clarity (Figure 7).

Regions of high strain in the $c$ axis corresponded to a relatively high proportion of hydroxyapatite crystallites/enamel prisms in the perpendicular angle population $\left(\sim 180^{\circ}\right.$, perpendicular to the cavity wall). At the top of the 'fin' where the strain is lowest ( - $0.0968 \%)$, approximately $75 \%$ of the hydroxyapatite crystallites belong to the perpendicular angle population whilst the remaining $25 \%$ belong to the non-perpendicular population $\left(\sim 210^{\circ}\right)$. In comparison, at the bottom of the 'fin' $\sim 90 \%$ of the crystallites are found in the perpendicular population which corresponds to the greatest magnitude 
of strain $(\sim-0.22 \%)$. This correlation is better visualised by a vertical line transect taken through the first column of data in this map (Figure 7) and plotted as the ratio of the magnitude of the $c$ axis strain to the perpendicular angle orientation population percentage as a function of track position (from top to bottom of the tooth 'fin') (Figure 8). Figure 8 demonstrates that $c$ axis strain increased with the percentage of crystallites oriented perpendicular to the cavity wall, showing a strong correlation of $r=$ 0.85. Strain in the $a$ axis (Figure 7c and d) did not show a clear spatial relationship with the orientation distribution.

\section{Discussion}

\subsection{Crystallographic Strain and Relief Mechanisms}

In this study we show that polymerisation of a dental composite within a tooth cavity can induce a measurable strain within the hydroxyapatite nano-crystals, that are the lowest hierarchical structural level of tooth enamel. Strain is due to stress transfer within the tooth structure as a consequence of shrinkage stresses generated by volumetric shrinkage of a constrained composite material. Previous laboratory based measurements using cuspal deflection techniques have to date only been able to correlate photo-polymerisation variables to the combined behaviour of all hierarchical levels of tooth enamel and dentine. Synchrotron XRD is the only technique that gives insight into the structure of hydroxyapatite nano-crystals over a relatively large region of interest. The results of this study show that the deformation of the unit cell within HAP nano-crystals is one mechanism by which strain can be detected, however others are known to exist and are likely acting simultaneously. Belchte et al showed that within the first hierarchical level, the protein matrix that binds HAP crystallites will demonstrate shear behaviour prior to strain being exhibited in the crystallite itself [70, 71]. Similarly, at the second structural level it was found that when loads were applied parallel to the $c$ axis of the enamel prisms that the stress / strain relationship was dictated predominantly by soft protein constituents, whilst at perpendicular loading stress / strain behaviour is governed by the crystal structure of the apatite. It is also possible, as demonstrated in similar systems, that the protein component may exist in a tensile state whilst the apatite phase is pre-compressed to store residual stress to protect against loading [72]. Additionally, Hon He et al reported that enamel has a stress-strain response similar to a 
metallic alloy and that backcreep (recovery) [38] is observed after a load is removed. It is therefore likely that the crystallographic strain observed in this study is not an absolute representation of the total strain generated by the shrinkage of the adhered composite with a portion of this being relieved via the protein matrix, or indeed micro-cracking within the enamel, induced by the creation of the measurement 'fin'. For meaningful 2D spatially resolved XRD measurements, thinning of the enamel was essential to avoid disproportionate averaging through the transmission thickness (i.e. to avoid the transmission thickness grossly exceeding beam-size dimensions). Machining will inevitably have some effect on the enamel structure and is unavoidable through this approach. Importantly our focus here was on studying relative changes in the machined enamel, using repeat measurements, which can be attributed to stresses generated by the constrained contraction of the composite. We accept that micro-cracking may act locally to relieve strain and have accordingly been careful to not over-generalize the findings.

\subsection{The Effect of Relative Irradiance on Crystallographic Strain}

In this study the irradiance of the LCU was used to produce relatively high and low assumed polymerisation rates. Polymerisation rate was not directly measured in this study, but studies have shown that the polymerisation rate is proportional to the intensity of the impinging light [73-75]. It can be seen in Figure 4 ( $\mathrm{c}$ and $\mathrm{f}$ ) that when the composite is photo-polymerised using higher irradiance (LED), that the hydroxyapatite crystallites demonstrate on average approximately $300 \%$ times greater strain in the crystallographic $c$ axis in comparison with the low light intensity regimen. First order differences in crystallographic strain for different light intensities are ascribed to the behaviour of the polymer phase of the composite as a function of reaction rate. Faster polymerisation advances the onset of gelation [65], a stage in which the mobility of the forming polymer decreases. Reduced mobility may inhibit elastic flow of the bulk resin matrix allowing greater internal stresses to build that will transfer to the composite-enamel interface. It must also be noted that although the low irradiance polymerisation regime shows negligible amounts of strain, this does not necessarily mean that no stress has been transferred to the crystalline structure, but relief mechanisms may have reduced this effect. 


\subsection{Spatial Distribution of Strain and Orientation}

Figure 4 demonstrates how crystallographic strain is spatially distributed in the tooth fin section for each photo-polymerisation regime. Strain for the low irradiance regime was close to being negligible; therefore the discussion and explanation of the spatial variation in strain will predominantly focus on the high irradiance effects. Strain in the $c$ axis was typically greatest towards the bottom of the fin and decreased gradually towards the top, although there were small regions that did not follow this pattern. This effect is attributed to the geometry of the mesio-occlusal-distal cavity following sample preparation. The remaining cusp acts as a cantilever beam when placed under load. Consequently, the cusp bends around a fixed point, producing maximum stress within the tooth towards the bottom of the cavity. In addition, the pattern of strain decreasing towards the cusp tip provided confidence that the changes in lengths of the $\mathrm{a}=\mathrm{b}$ and $\mathrm{c}$ axes were not due to small changes in sample to detector distance due to movement of the measurement fin over the course of the two measurement periods, as the converse pattern would be expected. A possible cause of sample movement, substrate dehydration was carefully considered. An initially hydrated tooth structure was required for application of dentine adhesive (required to allow the composite to bond to tooth structure). Following dentine adhesive application samples were left in air for a fixed time $>6 \mathrm{~h}$ prior to placement of composite and commencement of baseline and post-photo polymerization measurements. As this step would minimize dehydration rate, but not eliminate further dehydration during the overall measurement time we verified whether changes in the position of the 002 diffraction peak, occurred over a $4 \mathrm{~h}$ interval by taking additional track measurements during the 'post-cure' period. We found that the 002 peak positions for these two sets of measurements were practically identical, with the reported values within the errors of the fitting parameters. For example, the $2 \theta$ values for the 002 ring for the first positional measurement of track one, shown in Figure 5b, were $10.3165^{\circ}$ versus $10.3167^{\circ}+/-0.0005$ for the initial and repeated post cure measurements respectively.

It can be seen in Figure 4 that strain in the $c$ axis has a negative magnitude i.e. the axis is in a state of compression. This can potentially be ascribed to a Poisson contraction associated with cusp flexure. The measurement fin is located at the outer edge of the tooth (as shown by the blank map points along 
the XRD measurement tracks seen in Figure 4) and therefore will be in tension along its vertical axis as the cusp flexes. A corresponding contraction in the transverse direction, parallel to the $c$ axis, resulting in a decrease in the $c$ axis length is therefore feasible. This explanation also implies that the $a=b$ axis was placed under tension by this effect and that an increase in the axis length would be expected. However, here the $a=b$ axes did not increase and showed small negative values of strain in comparison to the $c$ axis, which are negligible for the low irradiance protocol. This suggests that relief mechanisms possibly at inter-prismatic, inter-rod or at higher hierarchical levels have reduced the measurable strain in the axis. It has been shown in dentin that the strain response may vary with axial direction due to differences in residual compressive stresses [72]. It is suggested that the $a$ axis may exist, over the measurement area, in a state of greater pre-existing residual compressive stress field relative to the $c$ axis and is therefore reduced strains arise following cusp flexure. Efficient strain relief mechanisms in this axis is unsurprising given the remarkable functional performance of enamel, which is subjected to severe cyclic loading over its lifetime.

Figure 3b shows that two directions of crystallite/enamel prism orientation exist, distributed about 180 and $210^{\circ}$ in the azimuthal plane. Typically, $75-90 \%$ of the crystallites ( $75-90 \%$ of the total integrated peak intensities, (Figure 3b)) display a preferred orientation about the perpendicular angle $\left(180^{\circ}\right)$ direction, which is almost normal to the cavity wall, although this varies spatially within each sample as seen in Figure 7. The perpendicular population is attributed to the HAP crystallites that comprise enamel prisms. This is based on its relatively large contribution to the total signal but also on the width of the peak. Hydroxyapatite crystals are not perfectly aligned parallel with neighbouring crystals nor to the long axis of the enamel prism but seem to entangle with each other [39] displaying a wide range of orientations. White et al (2001) [57] reported that peripheral nanorod hydroxyapatite crystals typically deviate by $\sim 60^{\circ}$ from the long axis of the nanorods to form a continuum with the inter-prismatic protein matrix. The non-perpendicular distribution is separated from the perpendicular distribution by $\sim 30^{\circ}$ which suggests that this distribution does not originate from the peripheral crystallites. It is likely that given the relatively large FWHM of the perpendicular distribution that the peripheral crystallites are included in this population. Additionally, Al-Jawad et al [54] showed that the crystallographic $c$ axis is 
typically oriented normal to the outer tooth surface which is in good agreement with an orientation angle of $\sim 180^{\circ}$. Alternatively, it is known that enamel prisms do not run continuously from the dental enamel junction to the outer enamel surface. Instead the inner section of enamel displays undulating prisms which may locally bend, twist and change their position [76], forming less common bands of decussating prisms. The presence of another orientation distribution (non-perpendicular angle) with a significantly different direction of preferred orientation and a lower contribution to the total diffraction signal suggests that this corresponds to decussated enamel prisms. Figure 6 shows that the azimuthal angle for both perpendicular and non-perpendicular angle distributions remained largely unaltered following photo-polymerisation. This suggests that whilst the presence of micro-cracks may play a role in altering local crystallite/prism orientation this role is likely minimal.

It can be seen in Figure 7 (a and b) that regions of greater strain in the $c$ axis correspond to a relatively high proportion of crystallites/prisms ( $90 \%$ of all crystallites/prisms) being oriented in the $180^{\circ}$ azimuthal direction. Conversely, lower $c$ strain was found to correlate to fewer crystallites/prisms oriented about $180^{\circ}$ (typically $\sim 75 \%$ ). Therefore, in regions of higher $c$ axis strain the average direction of crystallite orientation along the $c$ axis is approximately horizontal; being perpendicular to the cavity wall and parallel to the direction of contraction. Whereas in regions of lower strain the average orientation of the $c$ axis deviates from this horizontal geometry and is at a diagonal (in 2D) to the cavity wall, due to an increased proportion of crystallites/prisms in the non-perpendicular angle distribution. This suggests that the orientation of the $c$ axis relative to the bending moment of the cusp will affect the magnitude of strain that is observed. If it is assumed that a normal orientation of the $c$ axis relative to the cavity produces a maximum in strain, then if fewer crystals are oriented in this direction less strain would be detected. Furthermore, the high correlation between the proportion of perpendicularly oriented crystallites and observed strain strongly indicates that the perpendicular population isis exposed to greater strain than the non-perpendicular crystallites and that texture plays an important role in mediating strain in enamel. Additionally, regions where there is more $c$ axis alignment may be associated with greater macroscopic strain. However, these two effects cannot be dissociated.

There are some limitations to the experimental set-up in this study with respect to the light curing units used and the relationship between strain and monomer to polymer conversion. Two different LCUs 
were used to achieve relatively high and low light intensities. The LCUs have different emission spectra that will affect the production rate of radicals in addition to the light intensity of the respective LCU. Ideally these differences would be accounted for by normalising strain to monomer to polymer conversion. Unfortunately, the sample geometry and positioning did not allow for conversion measurements. We therefore accept uncertainties introduced by this, however due to the large difference in intensities between the two photo-polymerisation regimes and the associated differences in polymerisation rate we believe that overall trends in strain are statistically significant and may therefore be determined as a function of relative differences in the assumed polymerisation rate.

\section{Conclusions}

The spatial distribution of strain in human enamel following placement of a composite restoration has been studied ex vivo using high resolution synchrotron X-ray diffraction mapping, as a function of irradiance protocol which is proportional to the assumed polymerisation rate. Strain generated in the $c$ axis was greatest when a high irradiance protocol was used, which is proportional to an assumed increase in the polymerisation rate with respect to lower irradiances. This is in agreement with previously reported cuspal deflection methods. Negative values of strain were observed in the $c$ axis and are ascribed to cuspal flexure producing a Poisson's contraction, compressing the transverse axis, parallel to the c axis. However, strain in enamel is not uniformly distributed and varies spatially as a function of crystallite orientation. Greater alignment of crystallites perpendicular to the cavity wall was associated with higher $c$ axis strain. These findings give further insights which may explain the discrepancy between polymerisation variables, shrinkage stresses and the manifestation of enamel strain reported via conventional laboratory based techniques, providing a more complete understanding as to how composite polymerisation induces strain in the enamel.

\section{Acknowledgements}

This work was carried out with the support of the Diamond Light Source (proposal MT4016-1) on beamline B-16 with beamtime awarded through the Diamond Manchester Collaboration. 



\section{References}

1. Braga RR, Ferracane JL. Alternatives in polymerization contraction stress management. Crit Rev Oral Biol Med 2004;15(3):176-84.

2. Amirouche-Korichi A, Mouzali M, Watts DC. Effects of monomer ratios and highly radiopaque fillers on degree of conversion and shrinkage-strain of dental resin composites. Dent Mater 2009; 25(11):1411-1418.

3. Watts DC, Hindi AA. Intrinsic 'soft-start' polymerisation shrinkage-kinetics in an acrylatebased resin-composite. Dent Mater 1999;15(1):39-45.

4. Hofmann N, Hugo B, Klaiber B. Effect of irradiation type (LED or QTH) on photo-activated composite shrinkage strain kinetics, temperature rise, and hardness. Eur J Oral Sci 2002;110(6):471479.

5. Palin WM, Fleming GJ, Nathwani H, Burke FJ, Randall RC. In vitro cuspal deflection and microleakage of maxillary premolars restored with novel low-shrink dental composites. Dent Mater 2005;21(4):324-335.

6. Darvell BW. Chapter 5 - Acrylic. In: Darvell BW, editor. Materials Science for Dentistry, Woodhead Publishing; 2009, p.108-127.

7. Silikas N, Eliades G, Watts DC. Light intensity effects on resin-composite degree of conversion and shrinkage strain. Dent Mater 2000;16(4):292-296.

8. Stansbury JW, Trujillo-Lemon M, Lu H, Ding X, Lin Y, Ge J. Conversion-dependent shrinkage stress and strain in dental resins and composites. Dent Mater 2005;21(1):56-67.

9. Cara RR, Fleming GJ, Palin WM, Walmsley AD, Burke FJ. Cuspal deflection and microleakage in premolar teeth restored with resin-based composites with and without an intermediary flowable layer. J Dent 2007;35(6):482-489.

10. Moorthy A, Hogg CH, Dowling AH, Grufferty BF, Benetti AR, Fleming GJ. Cuspal deflection and microleakage in premolar teeth restored with bulk-fill flowable resin-based composite base materials. J Dent 2012;40(6):500-505.

11. Taha NA, Palamara JEA, Messer HH. Cuspal deflection, strain and microleakage of endodontically treated premolar teeth restored with direct resin composites. J Dent 2009;37(9):724-730. 
12. Kearns JO, Barry JG, Fleming GJ. Cuspal deflection and cervical microleakage scores to determine the adhesive potential of universal bonding systems. J Dent 2014;42(8):970-6.

13. Fleming GJP, Cara RR, Palin WM, Burke FJ. Cuspal movement and microleakage in premolar teeth restored with resin-based filling materials cured using a 'soft-start' polymerisation protocol. Dent Mater 2007;23(5):637-643.

14. Martin N, Jedynakiewicz NM, Williams DF. Cuspal deflection during polymerisation of composite lutes of ceramic inlays. J Dent 1999;27(1):29-36.

15. Alomari Q, Reinhardt JW, Boyer DB. Effect on cusp deflection and gap formation in composite restorations. Oper Dent 2001;26(4):406-11.

16. Li H, Li J, Yun X, Liu X, Fok AS. Non-destructive examination of interfacial debonding using acoustic emission. Dent Mater 2011;27(10):964-971.

17. Dauvillier BS, Aarnts MP, Feilzer AJ. Developments in Shrinkage Control of Adhesive Restoratives. J Esthet Restor Dent 2000;12(6):291-299.

18. Brunthaler A, König F, Lucas T, Sperr W, Schedle A. Longevity of direct resin composite restorations in posterior teeth. Clin Oral Investig 2003;7(2):63-70.

19. Davidson CL, Gee AJD, Feilzer A. The Competition between the Composite-Dentin Bond Strength and the Polymerization Contraction Stress. J Dent Res 1984;63(12):1396-1399.

20. Davidson CL, Feilzer AJ. Polymerization shrinkage and polymerization shrinkage stress in polymer-based restoratives. J Dent 1997; 25(6):435-440.

21. Soh MS, Yap AUJ, Sellinger A, Physicomechanical evaluation of low-shrinkage dental nanocomposites based on silsesquioxane cores. Eur J Oral Sci 2007;115(3):230-238.

22. Condon J.R, Ferracane J.L. Assessing the Effect of Composite Formulation on Polymerization Stress. J Am Dent Assoc 2000;131(4):497-503.

23. Feilzer AJ, Dauvillier BS. Effect of TEGDMA/BisGMA Ratio on Stress Development and Viscoelastic Properties of Experimental Two-paste Composites. J Dent Res 2003;82(10):824-828.

24. Davidson CL, de Gee AJ. Light-curing units, polymerization, and clinical implications. J Adhes Dent 2000;2(3):167-73. 
25. Uno S, Asmussen E. Marginal adaptation of a restorative resin polymerized at reduced rate. Eur J Oral Sci 1991;99(5):440-444.

26. Mehl A, Hickel R, Kunzelmann KH. Physical properties and gap formation of light-cured composites with and without 'softstart-polymerization'. J Dent 1997;25(3):321-330.

27. Hofmann N, Denner W, Hugo B, Klaiber B. The influence of plasma arc vs. halogen standard or soft-start irradiation on polymerization shrinkage kinetics of polymer matrix composites. J Dent 2003;31(6):383-393.

28. Sakaguchi RL, Berge HX. Reduced light energy density decreases post-gel contraction while maintaining degree of conversion in composites. J Dent 1998;26(8):695-700.

29. Feilzer AJ, Gee AJD, Davidson CL. Setting Stress in Composite Resin in Relation to Configuration of the Restoration. J Dent Res 1987;66(11):1636-1639.

30. Feilzer AJ, Gee AJD, Davidson CL. Increased Wall-to-Wall Curing Contraction in Thin Bonded Resin Layers. J Dent Res 1989;68(1):48-50.

31. Lee MR, Cho BH, Son HH, Um CM, Lee IB. Influence of cavity dimension and restoration methods on the cusp deflection of premolars in composite restoration. Dent Mater 2007;23(3):288-295. 32. Segura A, Donly KJ. In vitro posterior composite polymerization recovery following hygroscopic expansion. J Oral Rehabil 1993;20(5):495-499.

33. Meredith N, Setchell DJ. In vitro measurement of cuspal strain and displacement in composite restored teeth. J Dent 1997;25(3):331-337.

34. Pearson G, Hegarty SM. Cusp movement in molar teeth using dentine adhesives and composite filling materials. Biomaterials 1987;8(6):473-6.

35. Suliman AA, Boyer DB, Lakes RS. Interferometric Measurements of Cusp Deformation of Teeth Restored with Composites. J Dent Res 1993;72(11):1532-1536.

36. Alomari QD, Reinhardt JW, Boyer DB, Effect of liners on cusp deflection and gap formation in composite restorations. Oper Dent 2001;26(4):406-11.

37. Gwinnett AJ. Structure and composition of enamel. Oper Dent 1992;5;10-7.

38. He LH, Swain MV. Enamel-A "metallic-like" deformable biocomposite. J Dent 2007;35(5):431-437. 
39. Yilmaz ED, Schneider GA, Swain MV. Influence of structural hierarchy on the fracture behaviour of tooth enamel. Philos Trans A Math Phys Eng Sci 2015;373(2038).

40. Eastoe JE. Organic Matrix of Tooth Enamel. Nature 1960;187(4735):411-412.

41. Hu CC, Fukae M, Uchida T, Qian Q, Zhang CH, Ryu OH, Tanabe T, Yamakoshi Y, Murakami C, Dohi N, Shimizu M, Simmer JP. Sheathlin: Cloning, cDNA/Polypeptide Sequences, and Immunolocalization of Porcine Enamel Sheath Proteins. J Dent Res 1997;76(2):648-657.

42. He LH, Swain MV. Understanding the mechanical behaviour of human enamel from its structural and compositional characteristics. J Mech Behav Biomed Mater 2008;1(1):18-29.

43. Termine JD, Torchia DA, Conn KM, Enamel matrix: structural proteins. J Dent Res 1979;58(Spec Issue B):773-81.

44. Waltimo A, Könönen M, Maximal bite force and its association with signs and symptoms of craniomandibular disorders in young Finnish non-patients. Acta Odontol Scand 1995;53(4):254-258.

45. Hayasaki H, Okamoto A, Iwase Y, Yamasaki Y, Nakata M. Occlusal contact area of mandibular teeth during lateral excursion. Int J Prosthodont 2004;17(1):72-6.

46. Xu HHK, Smith DT, Jahanmir S, Romberg E, Kelly JR, Thompson VP, Rekow ED. Indentation Damage and Mechanical Properties of Human Enamel and Dentin. J Dent Res 1998;77(3):472-480.

47. Baldassarri M, Margolis HC, Beniash E. Compositional Determinants of Mechanical Properties of Enamel. J Dent Res 2008;87(7):645-649.

48. Schreger BNG, Beitrag zur Geschichte der Zähne. Beitrage für die Zergliederungskunst $1800 ; 1: 1-7$.

49. Osborn JW. The nature of the Hunter-Schreger bands in enamel. Arch Oral Biol, 1965. 10(6):929-IN30.

50. Paine ML, Snead ML. Protein Interactions During Assembly of the Enamel Organic Extracellular Matrix. J Bone Miner Res 1997;12(2):221-227.

51. Bartlett JD, Ganss B, Goldberg M, Moradian-Oldak J, Paine ML, Snead ML, Wen X, White SN, Zhou YL. Protein-Protein Interactions of the Developing Enamel Matrix. Curr Top Dev Biol 2006; 74:57-115. 
52. Deutsch D, Catalano-Sherman J, Dafni L, David S, Palmon A. Enamel Matrix Proteins and Ameloblast Biology. Connect Tissue Res 1995;32(1-4):97-107.

53. Simmons LM, Al-Jawad M, Kilcoyne SH, Wood DJ. Distribution of enamel crystallite orientation through an entire tooth crown studied using synchrotron X-ray diffraction. Eur J Oral Sci 2011;119 Suppl 1:19-24.

54. Al-Jawad M, Steuwer A, Kilcoyne SH, Shore RC, Cywinski R, Wood DJ. 2D mapping of texture and lattice parameters of dental enamel. Biomaterials 2007;28(18):2908-2914.

55. Simmons LM, Al-Jawad M, Kilcoyne SH, Wood DJ. Distribution of enamel crystallite orientation through an entire tooth crown studied using synchrotron x-ray diffraction. European Journal of Oral Sciences 2011;119:19-24

56. Spears IR, A three-dimensional finite element model of prismatic enamel: a re-appraisal of the data on the Young's modulus of enamel. J Dent Res 1997;76(10):1690-7.

57. White SN, Luo W, Paine ML, Fong H, Sarikaya M, Snead ML. Biological organization of hydroxyapatite crystallites into a fibrous continuum toughens and controls anisotropy in human enamel. J Dent Res 2001;80(1):321-6.

58. Xie Z, Swain MV, Hoffman MJ. Structural integrity of enamel: experimental and modelling. J Dent Res 2009;88(6):529-33.

59. He LH, Swain MV, Nanoindentation creep behavior of human enamel. J Biomed Mater Res A 2009;91(2):352-9.

60. Uno S, Asmussen E, Marginal adaptation of a restorative resin polymerized at reduced rate. Scand J Dent Res 1991;99(5):440-4.

61. Mehl A, Hickel R, Kunzelmann KH, Physical properties and gap formation of light-cured composites with and without 'softstart-polymerization'. J Dent 1997;25(3-4):321-30.

62. Sahafi A, Peutzfeldt A, Asmussen E, Soft-start polymerization and marginal gap formation in vitro. American J Dent 2001;14(3):145-7.

63. Miyazaki M, Oshida Y, Moore BK, Onose H. Effect of light exposure on fracture toughness and flexural strength of light-cured composites. Dent Mater 1996;12(6):328-32. 
64. Jantarat J, Panitvisai P, Palamara JE, Messer HH. Comparison of methods for measuring cuspal deformation in teeth. J Dent 2001; 29(1):75-82.

65. Braga RR., Ballester RY, Ferracane JL. Factors involved in the development of polymerization shrinkage stress in resin-composites: a systematic review. Dent Mater 2005;21(10):962-70.

66. Sawhney KJS, Dolbnya IP, Tiwari MK, Alianelli L, Scott SM, Preece GM, Pedersen UK, Walton RD. A Test Beamline on Diamond Light Source. AIP Conference Proceedings 2010;1234(1):387-390.

67. B16 Facility and Availability. http://www.diamond.ac.uk/Beamlines/Materials/B16/status.html. [accessed June 29 2017].

68. Hammersley AP, Svensson SO, Hanfland M, Fitch AN, Hausermann D. Two-dimensional detector software: From real detector to idealised image or two-theta scan. High Pressure Res 1996;14(4-6):235-248.

69. Siddiqui S, Anderson P, Al-Jawad M, Recovery of Crystallographic Texture in Remineralized Dental Enamel. PLoS One 2014;31;9(10).

70. Bechtle S, Fett T, Rizzi G, Habelitz S, Schneider GA. Mixed-mode stress intensity factors for kink cracks with finite kink length loaded in tension and bending: application to dentin and enamel. J Mech Behav Biomed Mater 2010;3(4):303-12.

71. Bechtle S, Habelitz S, Klocke A, Fett T, Schneider GA. The fracture behaviour of dental enamel. Biomaterials 2010;31(2):375-84.

72. Forein JB, Fleck C, Cloetens P, Duda G, Fratzl P, Zolotoyabko E, Zaslansky P. Compressive Residual Strains in Mineral Nanoparticles as a Possible Origin of Enhanced Crack Resistance in Human Tooth Dentin. Nano Lett 2015;15 (6):3729-3734.

73. Lovell LG, Newman SM, Bowman CN. The effects of light intensity, temperature, and comonomer composition on the polymerization behavior of dimethacrylate dental resins. J Dent Res 1999; 78:1469-1476.

74. Odian, G. Principles of polymerization. 3rd ed. New York: Wiley; 1991. 
75. Wydra JW, Cramer NB, Stansbury W, Bowman CN. The reciprocity law concerning light dose relationships applied to BisGMA/TEGDMA photopolymers: Theoretical analysis and experimental characterisation. Dent Mater 2014;30(6):605-12.

76. Radlanski R.J, Renz H, Willersinn U, Cordis CA, Duschner H. Outline and arrangement of enamel rods in human deciduous and permanent enamel. 3D-reconstructions obtained from CLSM and SEM images based on serial ground sections. Eur J Oral Sci 2001;109(6):409-14. 
Figure 1

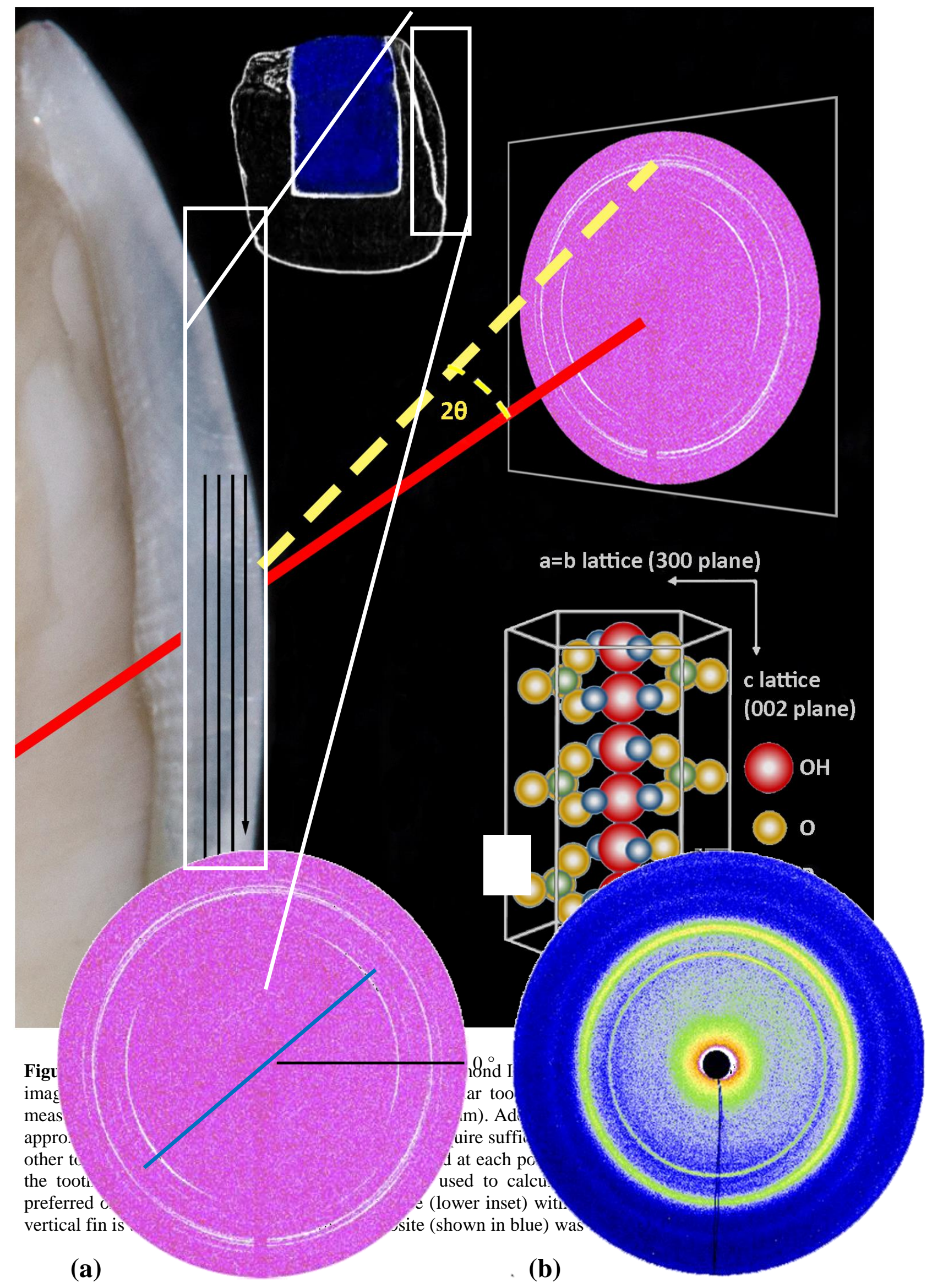




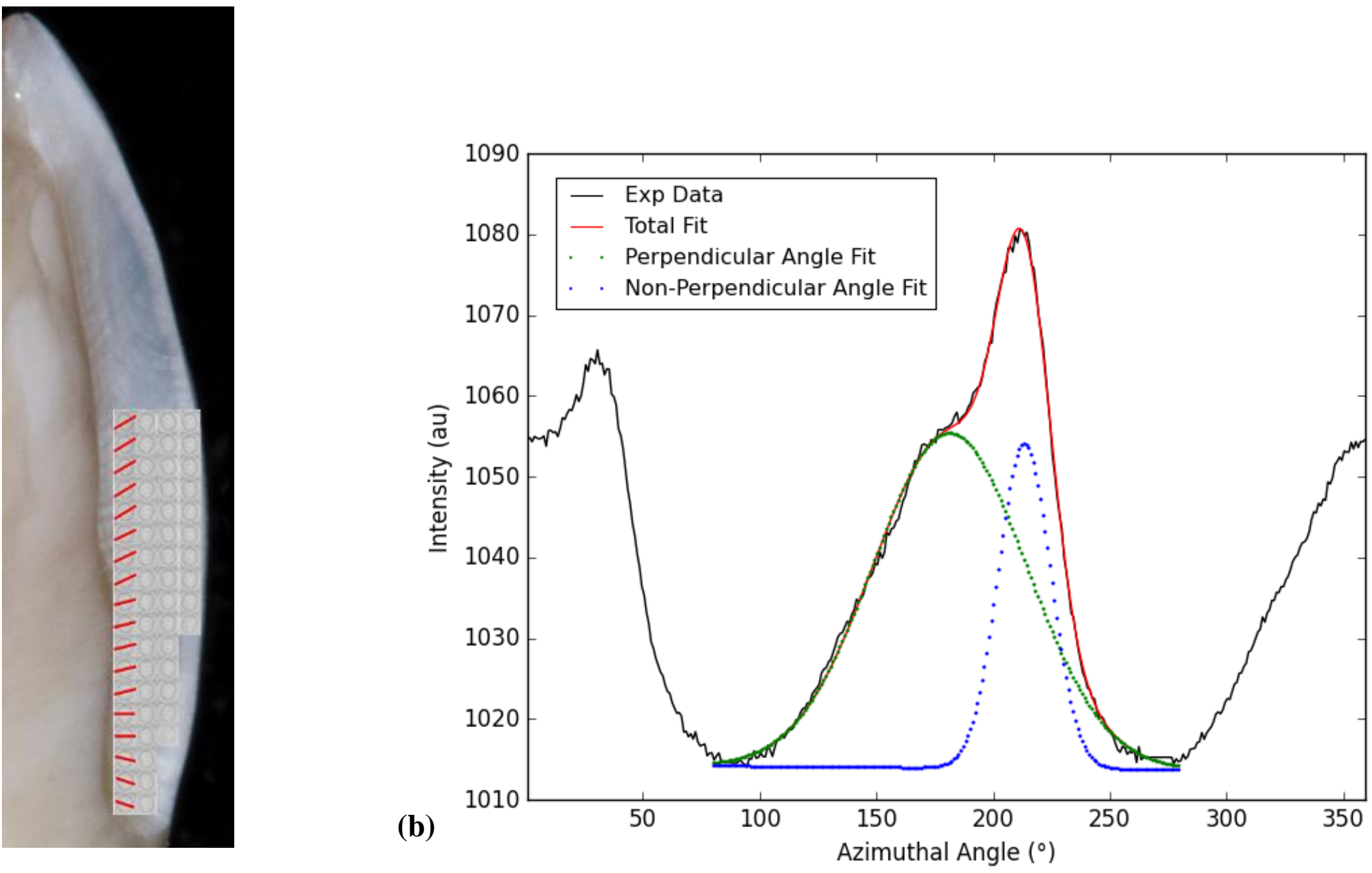

Figure 3. (a) A composite image showing individual 2-D diffraction patterns, for each measurement along a track (tracks 1-4, left to right), superimposed on to a schematic of a tooth enamel fin. Bisection of the (002) diffraction rings through points of greatest intensity yields the approximate direction of preferred orientation, which are shown as red lines. (b) Azimuthal integration of the (002) diffraction ring, followed by deconvolution, shows a bimodal distribution, indicating that two directions of crystallite/prismatic orientation exist within the tooth enamel. 
Figure 4

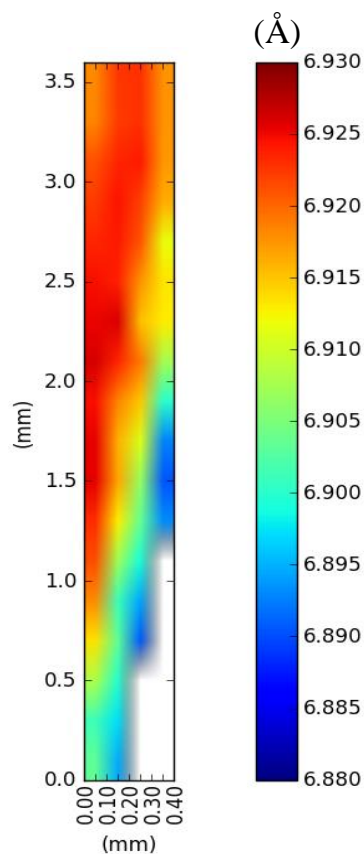

(a)

(̊)

(d)

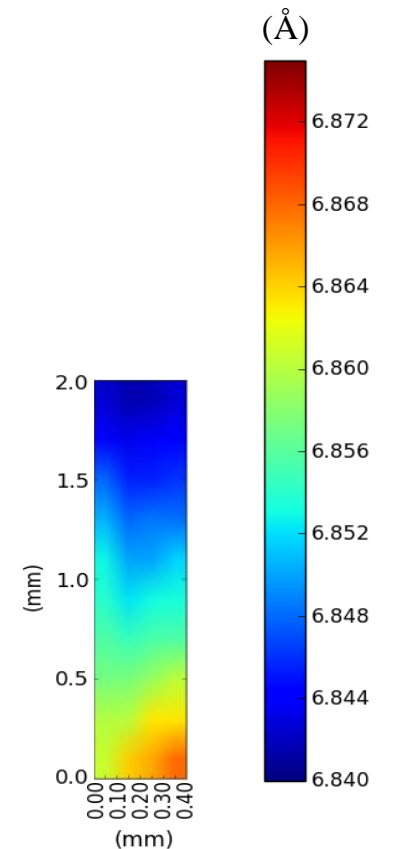

(b)

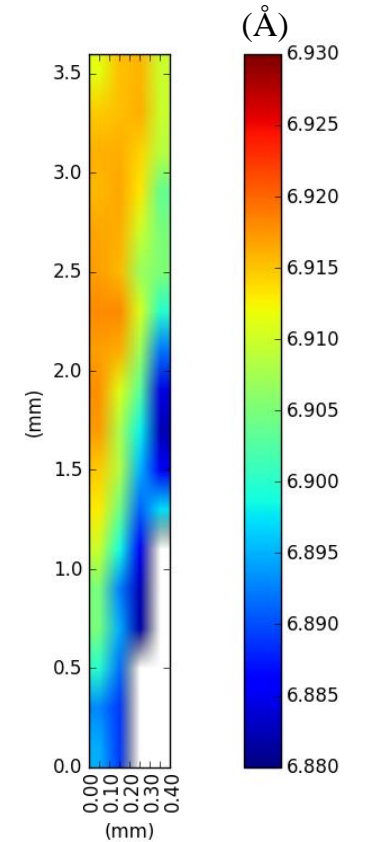

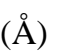

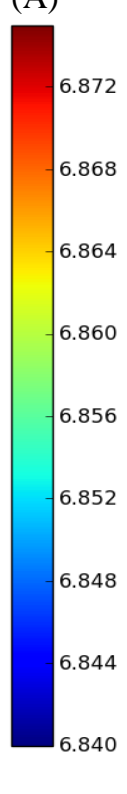

(c)

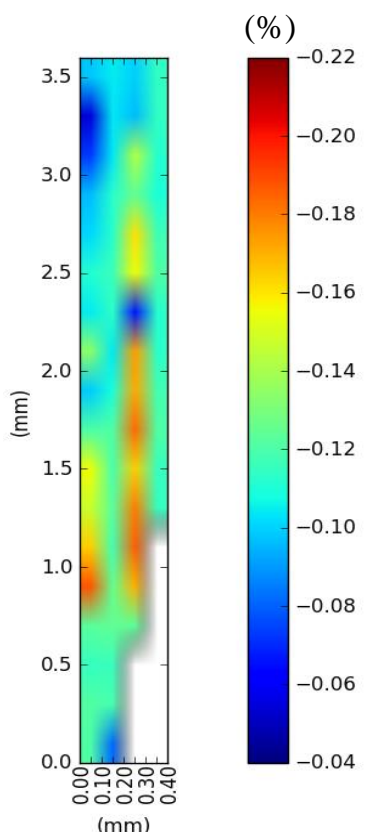

(\%)

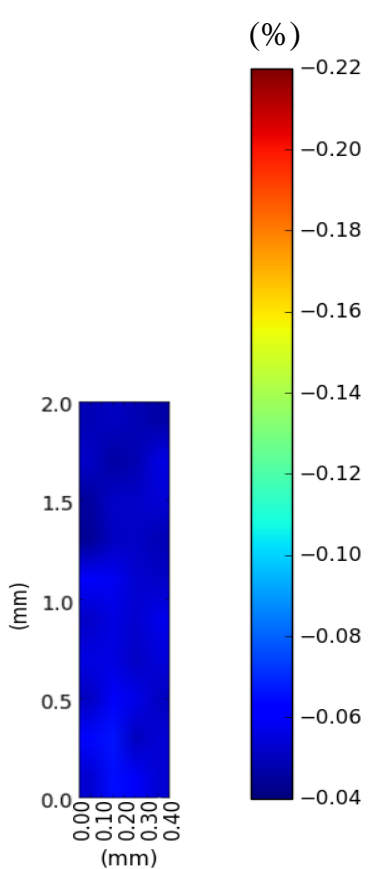

Figure 4. Contour maps for the $c$-lattice parameter over the scanned region of a given enamel 'fin' before and after LED and QTH curing. A colour scale-bar is shown to the right of each contour map. The cavity wall is located to the left of each contour map. (a) LED before. (b) LED after. (c) LED \% lattice strain; (d) QTH before. (e) QTH after .(f) QTH \% lattice strain. 

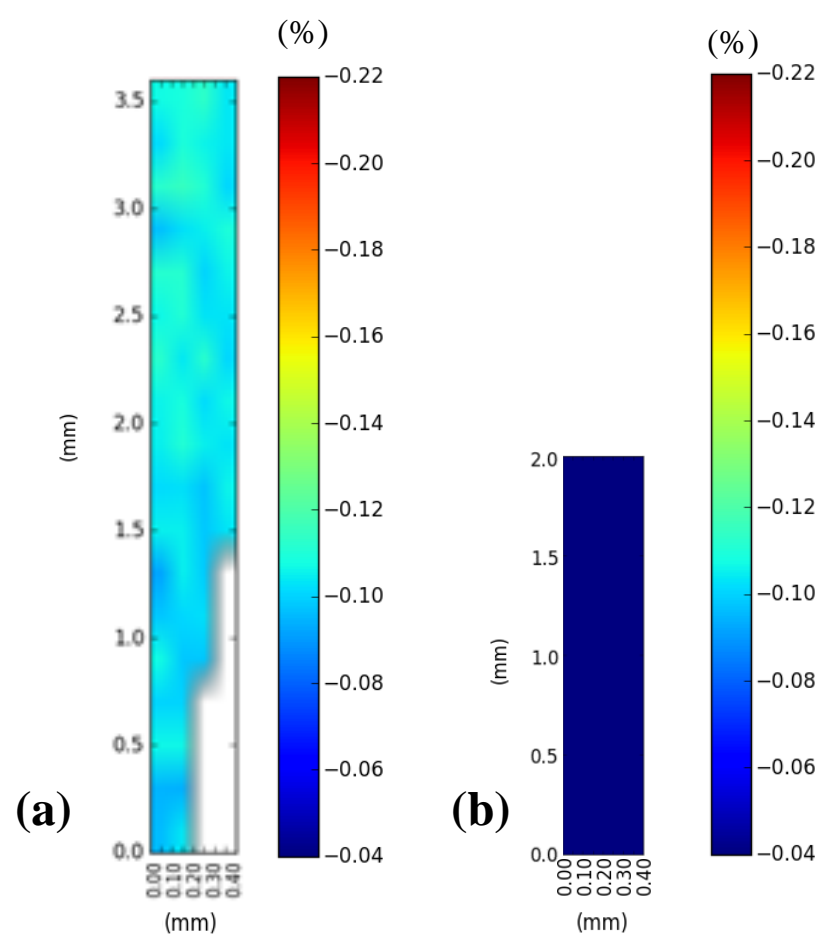

Figure 5. Crystallographic strain along the $a$ axis for (a) high and (b) low irradiance photopolymerization protocols.

\section{Figure 6}

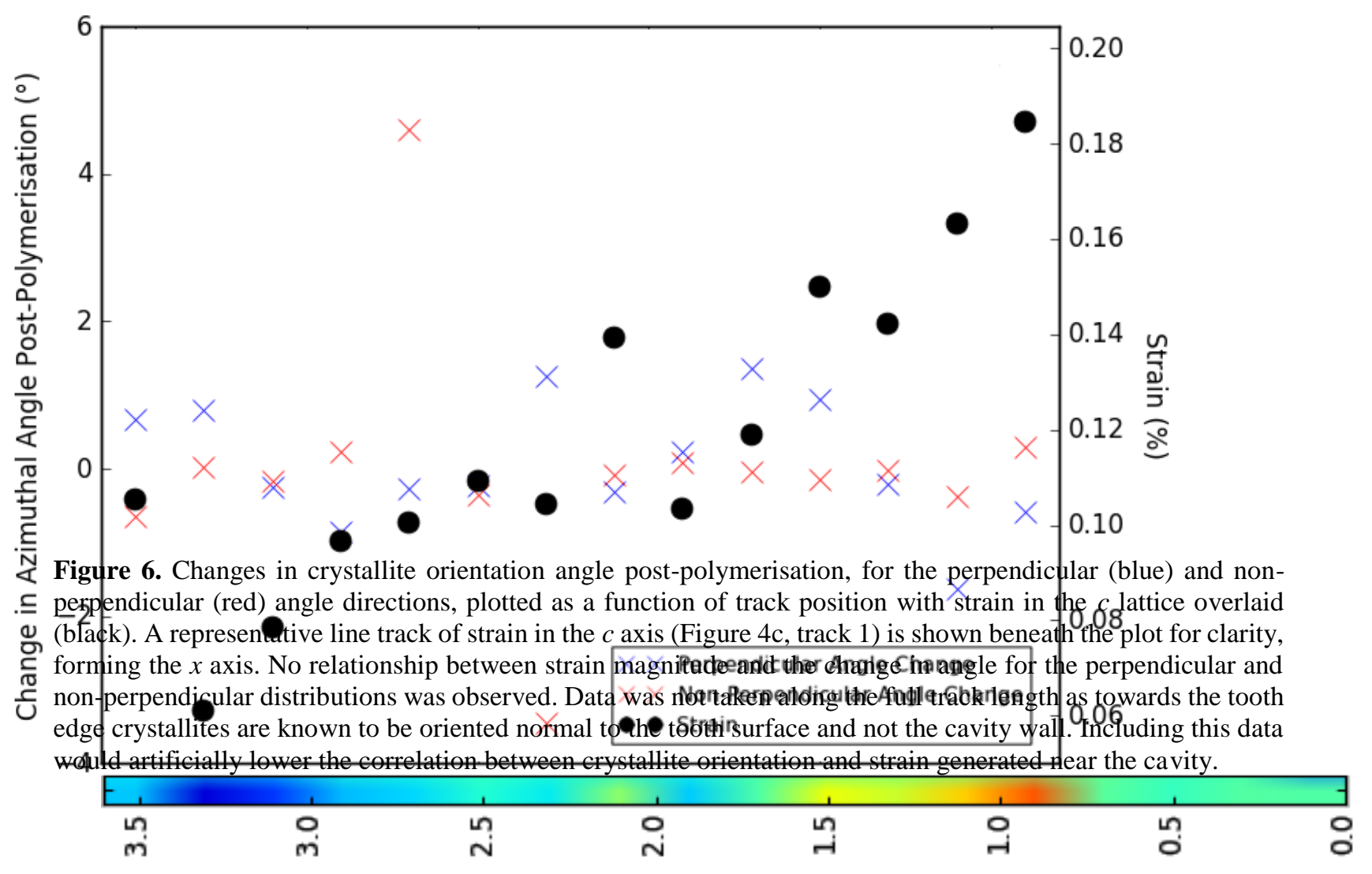


Figure 7

(\%)

(\%)

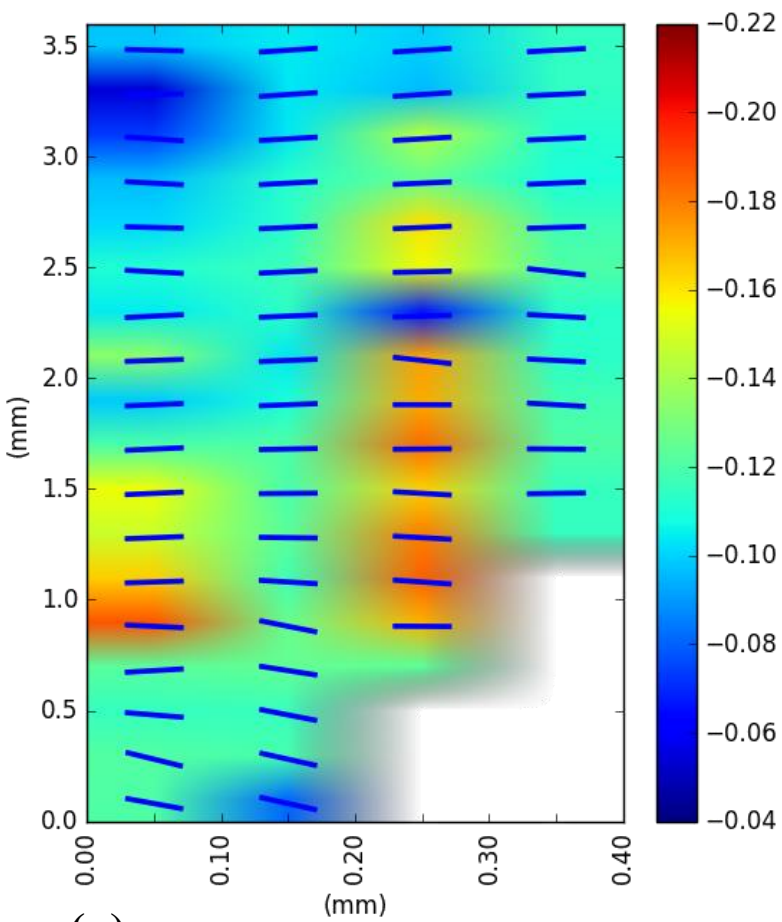

(a)

(\%)

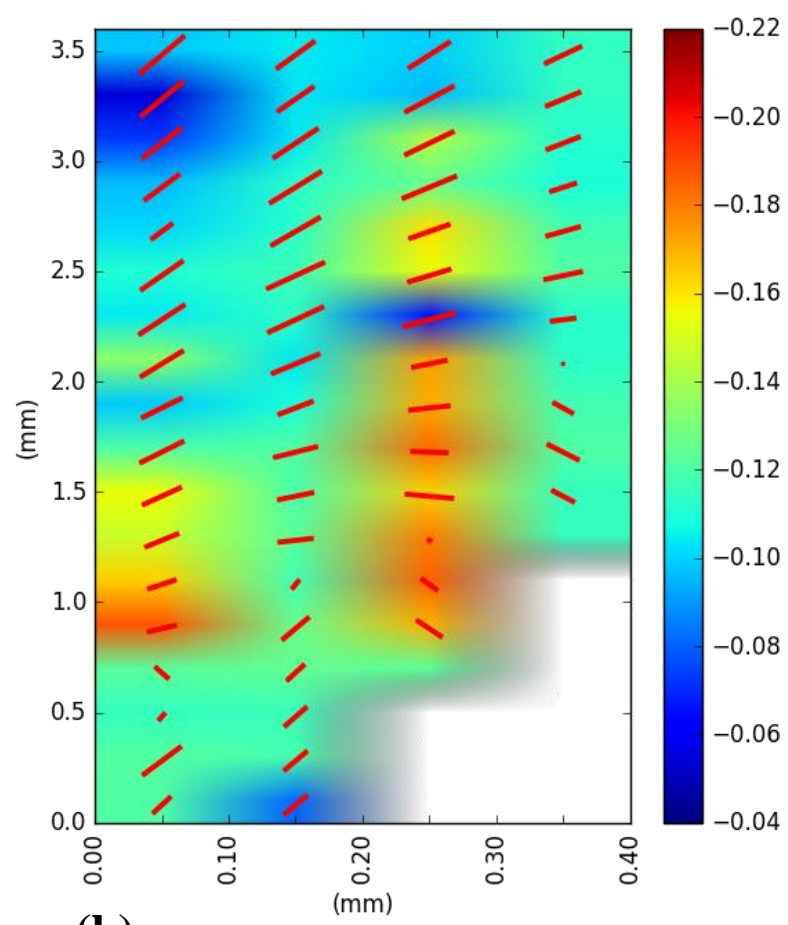

(b)

(\%)

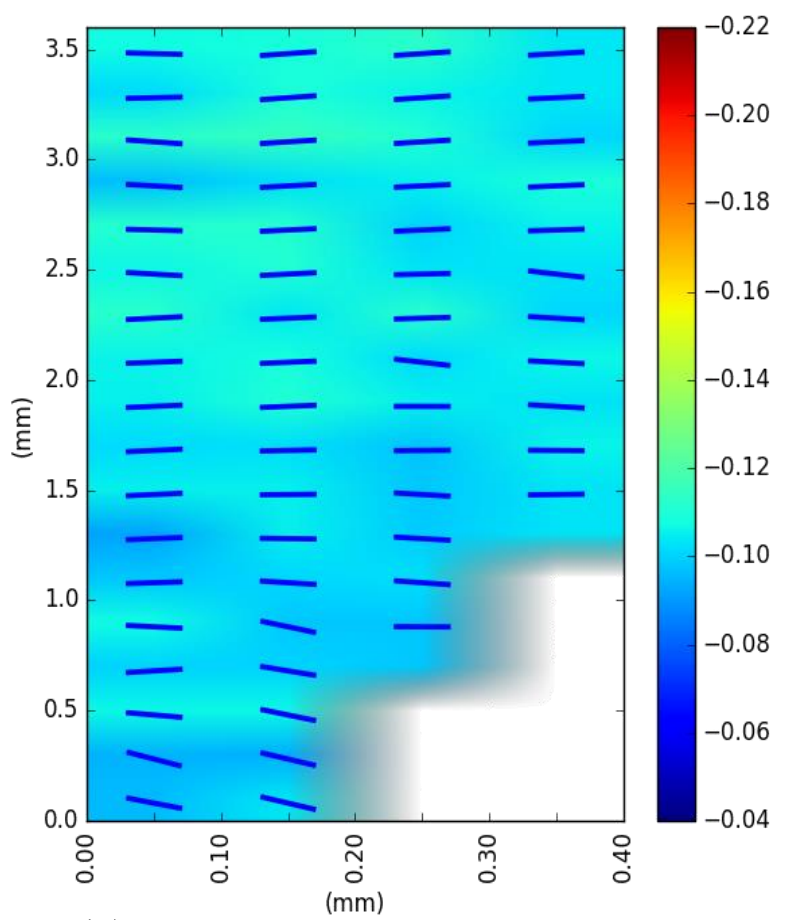

(c)

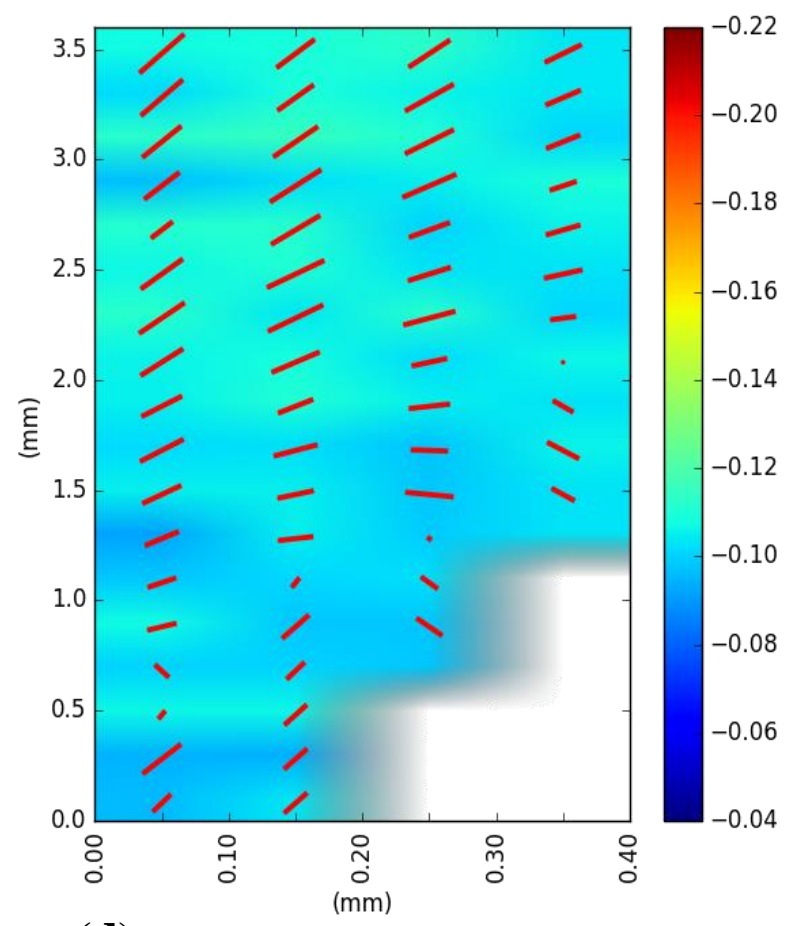

(d)

Figure 7. Orientation directions and the relative proportions of the perpendicular and non-perpendicular crystallite populations (blue and red bars respectively) overlaid on to strain data for the $c((\mathbf{a})$ and (b)) and $a$ axis ((c) and (d)). The red bars have been scaled in length by a factor of three and the aspect ratio of the underlying strain maps have been altered for clarity. 
Figure 8

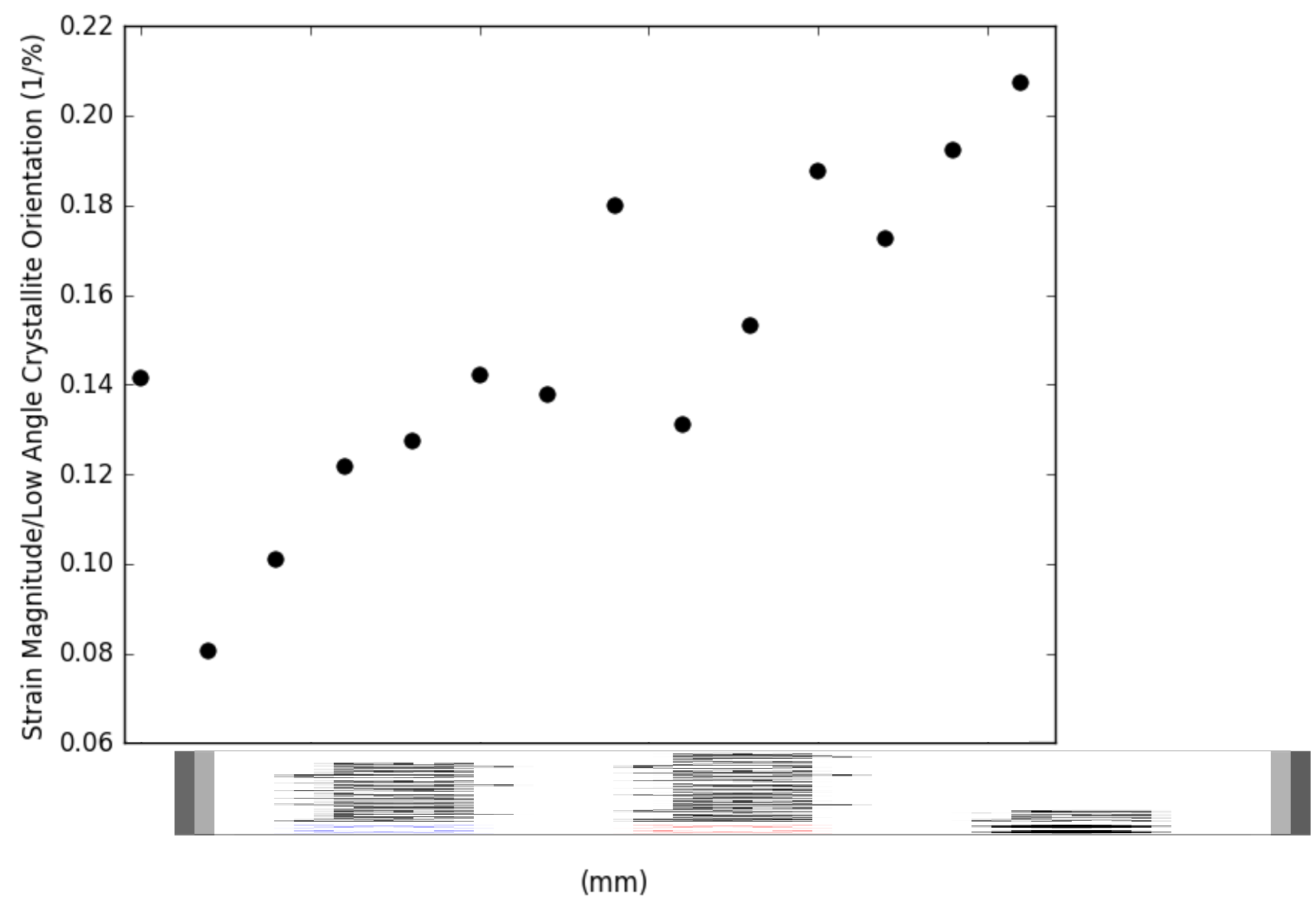

Figure 8. Ratio of the magnitude of the $c$ axis strain to the perpendicular angle orientation population percentage as a function of track position. A corresponding line track of strain in the $c$ axis is shown beneath the plot for clarity, forming the $x$ axis, taken from Figure 4c (track 1). Data was not taken along the full track length as towards the tooth edge crystallites are known to be oriented normal to the surface and not the cavity wall. Including this data would artificially lower the correlation between crystallite orientation and strain generated near the cavity. 